•编者按・

\title{
入侵科学的发展需要新视角和新技术
}

\author{
杨 继 ${ }^{*}$ 李 博
}

(生物多样性与生态工程教育部重点实验室, 上海崇明东滩湿地生态系统国家定位观测研究站,

上海崇明生态研究院, 复旦大学, 上海 200438)

\section{New perspectives and techniques are needed to advance invasion science}

\author{
Ji Yang ${ }^{*}$, Bo Li \\ Ministry of Education Key Laboratory for Biodiversity Science and Ecological Engineering, Shanghai Chongming \\ Dongtan Wetland Ecosystem Research Station, and Shanghai Institute of Eco-Chongming (SIEC), Fudan University, \\ Shanghai 200438
}

由人类活动导致的外来种入侵是全球性的生 物安全问题。随着全球化进程的加快, 外来入侵种 数量呈持续上升趋势(Essl et al, 2011; Seebens et al, 2017), 中国与世界各国都将面临更严峻的外来种 入侵威胁(Vaz et al, 2017)。为有效应对这一威胁, 各 国学者已对不同地区、不同类型生态系统中外来种 入侵的过程、生态学效应以及潜在驱动力等进行了 广泛的研究, 揭示了一系列与外来种入侵性 (invasiveness)以及生态系统可入侵性(invasibility)相 关联的影响因素, 并提出了一系列假说(如: 天敌逃 逸假说、竞争力增强的进化假说、空余生态位假说 等)用以解释外来种成功入侵的机制, 为外来入侵 种监测、管理和生态修复提供了依据。然而, 外来 种入侵是受多种因子驱动的、复杂的动态过程。很 多研究(如 Tecco et al, 2010)发现决定一个外来种能 否成功定殖和扩张的因素在不同地区或生态系统 中不尽相同，同一类型的生态系统对不同外来种的 易感性和抵御力也不一样, 每一种假说都有其应用 的局限性。因此, 要深入了解外来种成功入侵的机 理和规律, 仅仅研究已发生的入侵案例(研究特定 环境条件下的入侵案例) 是不够的, 需要针对特定 类群开展受控实验, 通过跟踪外来种对入侵地环境 因子变化的动态响应，探究入侵驱动因子及其作用 机制(Kueffer et al，2013)。在本专题中, 潘玉梅等 (2017) 比较了同质园中入侵种三叶鬼针草(Bidens pilosa $)$ 和大狼耙草 $(B$. frondos $a)$ 与本地种金盏银盘 (B. biternata)及狼耙草 (B. tripartita) 在不同光照和水 分交互作用下的生长表现, 发现入侵种对光照响应 的可塑性指数大于本地种。入侵种较强的表型可塑 性有助于提高其捕获和利用资源的能力, 并促进其 入侵。王家宜等(2017)比较了入侵种薇甘菊(Mikania micrantha) 与本地植物粉葛 (Pueraria lobata var. thomsonii) 对干旱及涝害胁迫的生理响应差异, 结 果显示尽管薇甘菊在入侵生境中生长与繁殖力极 强, 入侵扩散速度极快, 但对水分胁迫的抗逆性低 于粉葛。这一结果为开发替代控制技术提供了依据。

本质上, 外来种入侵性的表达体现为其决定入 侵性的功能性状与土著生态系统中功能群相互作 用, 从而改变生态系统结构和功能的过程(Vilà et al, 2011)。研究外来种入侵性与生态系统可入侵性的关 系, 实际上就是要探明关键功能性状在局域尺度环 境中特定的表达式样以及不同功能性状间的内在 联系和权衡关系。由于外来种在不同入侵生境中、 在不同入侵阶段面临不同的选择压力, 其功能性状 的作用及其与环境的互作关系均表现出动态变化 的特点。以功能性状分析为基础, 把入侵性表达、 功能群替代与生态系统过程连接起来, 从生态网络 角度解析外来生物入侵性与生境脆弱性、生态系统 可入侵性的关系, 是揭示外来种入侵生态机制和成 灾机理的关键, 也是入侵生物学研究的发展趋势 
(Hui et al, 2016)。在本专题中，付伟等(2017)聚焦于 土壤微生物与入侵植物之间相互作用及其对入侵 性的影响, 指出植物根际为微生物提供了多样化的 异质环境(根际土壤、根表和内生环境), 并塑造了其 功能多样性; 建议在借助快速发展的高通量测序技 术拓展研究深度和广度的同时, 要关注根际微生境 与宏观功能特性的结合与统一。周方等(2017)比较 了喜旱莲子草(Alternanthera philoxeroides)原产地和 入侵地种群在不同养分条件下，与防御相关的功能 性状(比茎长，根冠比)及其对天敌取食的诱导反应, 发现入侵地种群对专食性天敌的组成抗性低于原 产地种群。此外, 入侵地种群对专食性天敌的诱导 抗性尽管在低养分条件下低于原产地种群，但在高 养分条件下高于原产地种群, 表明生境的养分富集 可能会降低专食性天敌对喜旱莲子草的生物防治 效果。

全球气候变化背景下, 生态系统内不同功能群 的差别响应可能改变彼此间的互作模式, 进而影响 入侵过程和态势。孙燕等(2017)通过预测豚草 (Ambrosia artemisiifolia)及豚草条纹叶甲(Ophraella communa) 和豚草卷蛾 (Epiblema strenuana)未来的适 宜分布区, 发现气候变化可能会导致两种生物防治 天敌(尤其是豚草条纹叶甲)与豚草的地理分布重叠 区域减少，进而影响生物防治效果。

尽管围绕生物入侵机制、影响及其管控策略的 研究已取得诸多重要成果和进展, 但仍有一些基本 的问题尚未解决。例如，长期演化形成的生物地理 分布格局如何影响生态系统对外来生物入侵的易 感性和抵御力? 为什么外来物种中只有少部分发 展成为恶性入侵种? 适应性进化与外来种成功入 侵有何关系? 如何权衡外来种管控与经济发展及 社会文化的关系等(Richardson, 2011; Kueffer et al,
2013)。外来种在全球范围的分布区扩张是人类世 (anthropocene)的一个显著特征，这一趋势在很大程 度上归因于全球化贸易和交通运输网络的发展。因 此, 外来种入侵机理及其生态和经济影响分析不仅 是制订科学管理和发展策略的基础，而且能帮助理 解为什么我们正从以具有丰富而独特生物地理区 为特征的全新世(holocene)向同质化的、以多样性丧 失为特征的人类世发展 (Lewis \& Maslin，2015; Wilson et al, 2016); 外来种入侵研究也不再局限于 生态学或生物学层面, 而需要从社会一生态系统的 角度加以分析和认识(Richardson, 2015; Vaz et al, 2017)。我们不仅仅要关注外来种分布区扩张对自然 生物地理分布区、对生态系统的影响，而且要分析 外来种入侵的社会动因, 分析外来种与经济、文化 以及价值观发展的联系，分析人类社会系统与自然 生态系统的耦合关系(Essl et al, 2017; Vaz et al, 2017)。在着力维护生态系统结构和功能的完整性使 之免受外来种入侵影响的同时，我们也要接受被改 变的生态系统，并致力于促进其发生健康的变化， 而不总是试图让生态系统恢复过去的状态 (Barnosky et al, 2017)。处在从入侵生态学(invasion ecology)、入侵生物学(invasion biology) 向入侵科学 (invasion science)跨越发展的时代，我们仍然面临 诸多挑战，但新技术的发展也为我们更深入地研究 外来生物的入侵机理、更有效地监测和控制提供了 机遇和条件(Ricciardi et al, 2017)。

本文中所引用的文献参见附录 1 (http://www.biodiversity-science.net/fileup/PDF/2018007-1.pdf)。

(责任编辑: 周玉荣) 


\section{附录1 参考文献}

Barnosky AD, Hadly EA, Gonzalez P, Head J, Polly PD, Lawing AM, Eronen JT, Ackerly DD, Alex K, Biber E, Blois J, Brashares J, Ceballos G, Davis E, Dietl GP, Dirzo R, Doremus H, Fortelius M, Greene HW, Hellmann J, Hickler T, Jackson ST, Kemp M, Koch P, Kremen C, Lindsey EL, Looy C, Marshall CR, Mendenhall C, Mulch A, Mychajliw AM, Nowak C, Ramakrishnan U, Schnitzler J, Shrestha KD, Solari K, Stegner L, Stegner MA, Stenseth NC, Wake MH, Zhang Z (2017) Merging paleobiology with conservation biology to guide the future of terrestrial ecosystems. Science, 355, eaah4787.

Essl F, Dullinger S, Rabitsch W, Hulme PE, Hülber K, Jarošík V, Kleinbauer I, Krausmann F, Kühn I, Nentwig W, Vilà M, Genovesi P, Gherardi F, Desprez-Loustau ML, Roques A, Pyšek P (2011) Socioeconomic legacy yields an invasion debt. Proceedings of the National Academy of Sciences, USA, 108, 203-207.

Essl F, Hulme PE, Jeschke JM, Keller R, Pyšek P, Richardson DM, Saul WC, Bacher S, Dullinger S, Estévez RA, Kueffer C, Roy HE, Seebens H, Rabitsch W (2017) Scientific and normative foundations for the valuation of alien-species impacts: thirteen core principles. BioScience, 67, 1-13.

Fu W, Wang N, Pang F, Huang YL, Wu J, Qi SS, Dai ZC, Du DL (2017) Soil microbiota and plant invasions: current and future trends. Biodiversity Science, 25, 1295-1302. (in Chinese with English abstract) [付伟，王宁，庞芳，黄玉龙，吴俊， 祁珊珊, 戴志聪, 杜道林 (2017) 土壤微生物与植物入侵: 现状与未来. 生物多样性, 25, 1295-1302.]

Hui C, Richardson DM, Landi P, Minoarivelo HO, Garnas J, Roy HE (2016) Defining invasiveness and invasibility in ecological networks. Biological Invasions, 18, 971-983.

Kueffer C, Pyšek P, Richardson DM (2013) Integrative invasion science: model systems, multi-site studies, focused meta-analysis and invasion syndromes. New Phytologist, 200, 615-633.

Lewis SL, Maslin MA (2015) Defining the anthropocene. Nature, 519, 171-180.

Pan YM, Tang SC, Wei CQ, Li XQ (2017) Comparison of growth, photosynthesis and phenotypic plasticity between invasive and native Bidens species under different light and water conditions. Biodiversity Science, 25, 1257-1266. (in Chinese with English abstract) [潘玉梅, 唐赛春, 韦春强, 李象钦 (2017) 不同光照和水分条件下鬼针草属入侵种与 本地种生长、光合特征及表型可塑性的比较. 生物多样性, 25, 1257-1266.]

Ricciardi A, Blackburn TM, Carlton JT, Dick JT, Hulme PE, Iacarella JC, Jeschke JM, Liebhold AM, Lockwood JL, Macisaac HJ, Pyšek P, Richardson DM, Ruiz GM, Simberloff D, Sutherland WJ, Wardle DA, Aldridge DC (2017) Invasion science: a horizon scan of emerging challenges and opportunities. Trends in Ecology \& Evolution, 32, 464-474.

Richardson D (2015) Biological invasions \& the emergence of invasion science: cover story. Quest, 11, 5-7.

Richardson DM (2011) Invasion science: the roads travelled and the roads ahead. Fifty Years of Invasion Ecology: The Legacy of Charles Elton, 397-401.

Seebens H, Blackburn TM, Dyer EE, Genovesi P, Hulme PE, Jeschke JM, Pagad S, Pyšek P, Winter M, Arianoutsou M, with other 35 co-authors (2017) No saturation in the accumulation of alien species worldwide. Nature Communications, 8, 14435.

Sun Y, Zhou ZS, Wang R, Müller-Schärer H (2017) Biological control opportunities of ragweed are predicted to decrease with climate change in East Asia. Biodiversity Science, 25, 1285-1294. (in Chinese with English abstract) [孙燕, 周忠 实, 王瑞, Müller-Schärer H (2017) 气候变化预计会减少东 亚地区豚草的生物防治效果。生物多样性, 25 , 1285-1294.]

Tecco PA, Díaz S, Cabido M, Urcelay C (2010) Functional traits of alien plants across contrasting climatic and land-use regimes: do aliens join the locals or try harder than them? Journal of Ecology, 98, 17-27.

Vaz AS, Kueffer C, Kull CA, Richardson DM, Schindler S, Muñoz-Pajares AJ, Vicente JR, Martins J, Hui C, Kühn I (2017) The progress of interdisciplinarity in invasion science. Ambio, 46, 428.

Vilà M, Espinar JL, Hejda M, Hulme PE, Jarošík V, Maron JL, Pergl J, Schaffner U, Sun Y, Pyšek P (2011) Ecological impacts of invasive alien plants: a meta-analysis of their effects on species, communities and ecosystems. Ecology Letters, 14, 702-708.

Wang JY, Yu HX, Lai YF, Wan FH, Qian WQ, Peng CL, Li WH (2017) Physiological response of the invasive weed Mikania micrantha and the native species Pueraria lobata var. thomsonii to water stress. Biodiversity Science, 25, 1267-1275. (in Chinese with English abstract) [王家宜, 余 涵霞, 赖玉芳, 万方浩, 钱万强, 彭长连, 李伟华 (2017) 入侵杂草薇甘菊与本地植物粉葛对水分胁迫的生理响应. 生物多样性, 25, 1267-1275.]

Wilson JRU, García-Díaz P, Cassey P, Richardson DM, Pyšek P, Blackburn TM (2016) Biological invasions and natural colonisations are different - the need for invasion science. NeoBiota, 31, 87.

Zhou F, Zhang ZJ, Liu M, Pan XY (2017) Effects of nutrient levels on defense against specialist insects in an invasive alligator weed. Biodiversity Science, 25, 1276-1284. (in Chinese with English abstract) [周方, 张致杰, 刘木, 潘晓 云 (2017) 养分影响入侵种喜早莲子草对专食性天敌的防 御. 生物多样性, 25, 1276-1284.] 